\title{
The association of neck circumference with incident congestive heart failure and coronary heart disease mortality in a community-based population with or without sleep-disordered breathing
}

\author{
Jingjing Zhang ${ }^{1 \dagger}$, Qi Guo ${ }^{2 \dagger}$, Liyuan Peng ${ }^{3}$, Jiamei Li ${ }^{1}$, Ya Gao ${ }^{1}$, Bin Yan , Bangjiang Fang ${ }^{5}$ and Gang Wang ${ }^{1 *}$ (i)
}

\begin{abstract}
Background: Neck circumference (NC), representing upper body subcutaneous adipose tissue, may be correlated with increased risk of overweight/obesity, obstructive sleep apnoea, and metabolic and cardiovascular disease. However, the relationship between NC and the incidence of congestive heart failure (CHF) or mortality due to coronary heart disease (CHD) in a community-based population with and without sleep-disordered breathing (SDB) has not yet been clarified.

Methods: We performed a prospective study using the Sleep Heart Health Study (SHHS) cohort. Cox proportional hazards regression models were used to estimate the association of different levels of NC with CHF incidence or CHD mortality in 2234 individuals with SDB and 2199 without SDB, respectively.

Results: After adjusting for age, sex, and body mass index (BMI), NC was significantly associated with CHF when comparing the highest NC quartile group with the lowest (hazard ratio, HR, 2.265, 95\% confidence interval, Cl, 1.074-4.777) in the non-SDB population. This association diminished after further adjustment for other risk factors, but remained statistically significant, with an adjusted HR of 1.082 (95\% Cl 1.003-1.166) per unit increase in NC. Additionally, after adjustment for age, sex, and BMI, NC was also shown to be remarkably associated with CHD mortality (HR 1.141,95\% Cl 1.014-1.282) per unit increase in NC in the non-SDB population but not in the SDB population. After adjustment for all the covariates, there was a significant association between NC and CHD death in those without SDB, with an adjusted HR of 1.134 (95\% Cl 1.001-1.284) per unit increase in NC.
\end{abstract}

Conclusions: NC may correlate with CHF incidence and CHD mortality in population without SDB. NC measurement may help risk stratification for cardiovascular diseases.

Trial registration: NCT00005275, January 1994.

Keywords: Neck circumference, Congestive heart failure, Coronary heart disease, Mortality,

Sleep-disordered breathing

\footnotetext{
* Correspondence: gang_wang@mail.xjtu.edu.cn

†Jingjing Zhang and Qi Guo contributed equally to this work.

${ }^{1}$ Department of Emergency Medicine, the Second Affiliated Hospital of Xi'an

Jiaotong University, Xi'an 710004, China

Full list of author information is available at the end of the article
}

(c) The Author(s). 2018 Open Access This article is distributed under the terms of the Creative Commons Attribution 4.0 International License (http://creativecommons.org/licenses/by/4.0/), which permits unrestricted use, distribution, and reproduction in any medium, provided you give appropriate credit to the original author(s) and the source, provide a link to the Creative Commons license, and indicate if changes were made. The Creative Commons Public Domain Dedication waiver (http://creativecommons.org/publicdomain/zero/1.0/) applies to the data made available in this article, unless otherwise stated. 


\section{Background}

Obesity, a pathological condition with body mass index (BMI) over $30 \mathrm{~kg} / \mathrm{m}^{2}$, is associated with a series of metabolic risk factors [1]. However, the overall obesity includes unique intrinsic characteristics of different fat depots and the pattern of body fat distribution may also contribute to the prognosis [2]. Recently, significant heterogeneity was found between visceral adipose tissue (VAT) and subcutaneous adipose tissue (SAT) of the upper body. VAT is recognized as a pathogenic fat deposit with increased risk for insulin resistance, type II diabetes mellitus, and atherosclerosis [3, 4]. It was found that while lower body SAT may reduce the risk for cardiovascular disease, abdominal SAT was not related [5].

Previous studies have demonstrated that upper body fat produced the majority of systemic free fatty acids (FFA), which was associated with insulin resistance, increased very low-density lipoprotein, and endothelial dysfunction [3]. Neck circumference (NC), measured at the level of the laryngeal prominence, represents upper body SAT and may be correlated with increased risk of overweight/obesity, obstructive sleep apnoea, type II diabetes, metabolic syndrome, and cardiovascular disease [3, 4, 6-10]. However, the relationship between NC and the incidence of congestive heart failure (CHF) or mortality due to coronary heart disease (CHD) has not yet been clarified. Previously, the Sleep Heart Health Study (SHHS) was carried out in a geographically diverse, community-based population to assess the difference in cardiovascular outcomes among participants with different sleep apnoea statuses [11]. Based on SHHS, the present study aimed to investigate the role of $\mathrm{NC}$ in the incidence of CHF or CHD mortality in populations with and without sleep-disordered breathing (SDB).

\section{Methods}

\section{Study design}

Analyses were carried out in two subsets of data obtained from the participants of SHHS (ClinicalTrials.gov Identifier: NCT00005275). A total of 5804 participants aged 40 years and older underwent home polysomnography and completed a set of questionnaires on general health and sleep habits since 1994 [12]. Cardiovascular disease outcomes were tracked until 2010. Detailed aims and design of SHHS are described elsewhere [11]. The protocol was approved by the Institutional Review Board of each participating institution and signed informed consents were provided by the subjects. The data were accessed based on a signed agreement with the Brigham and Women's Hospital.

\section{Participants}

A total of 5804 subjects from SHHS cohort included 1915 from the Atherosclerosis Risk in Communities study, 1230 from the Cardiovascular Health Study, 688 from Framingham Offspring Cohort, and 1971 from other studies. Of them, 600 subjects were excluded because of pre-existing CHD, heart failure, or other related cardiovascular events, 762 because of lack of follow-up data, and 9 because of lack of NC data, ultimately leaving an analytical sample size of 4433. SDB has been proposed as a risk factor for cardiovascular events; besides, SHHS mainly focused on the relationship between SDB and cardiovascular outcomes. Therefore, our investigation was carried out in the following two groups: the group with SDB and the group without SDB (Additional file 1: Figure S1).

\section{$\mathrm{NC}$ and SDB}

$\mathrm{NC}$ was measured during health interviews at the beginning of the observation period. Participants were advised to sit upright and look straight ahead. An inelastic tape was applied around the neck just below the laryngeal prominence. The NC measurement was made perpendicular to the long axis of the neck with the tape contacting the skin surface under acceptable pressure. Subjects were divided into $\mathrm{NC}$ quartiles of the following ranges: $\leq 34.1$ (reference), 34.1-37.0, 37.0-40.5, and $>40.5 \mathrm{~cm}$ [13]. Home polysomnography was performed with the Compumedics P-series portable monitor (Abbotsford, Victoria, Australia). Detailed information were collected as described in a previously published work [14]. Apnoea was defined as complete or almost complete cessation of airflow lasting for at least $10 \mathrm{~s}$, while hypopnea was defined as a clearly discernible decrease in airflow, or chest or abdominal plethysmograph amplitude that lasted for at least $10 \mathrm{~s}$. Both, apnoea and hypopnea, required an associated $4 \%$ or greater oxyhaemoglobin desaturation. $\mathrm{AHI}$ is the average number of episodes of apnoea and/ or hypopnea per hour of sleep [15]. SDB was defined as $\mathrm{AHI} \geq 5$ respiratory events per hour while non-SDB was defined as $\mathrm{AHI}<5$.

\section{CHD death and CHF}

Baseline CHD or CHF was defined to be present if the participant responded positively to a standardized questionnaire before the polysomnogram monitoring, or reported that they had undergone coronary bypass surgery or coronary angioplasty, or if the parent cohort had an identified CHD event or CHF before the SHHS baseline. CHD death was defined as fatal CHD at any time between the baseline polysomnogram and the final follow-up between 2008 and 2011. Incident CHF was defined as the first occurrence of heart failure during this period of follow-up. Ongoing surveillance for $\mathrm{CHD}$ deaths and incident $\mathrm{CHF}$ events was carried out by the parent cohorts according to cohort-specific protocols. 


\section{Other covariates}

Smoking status was classified as "never" (if the participant reported lifetime smoking of fewer than 20 packs of cigarettes), "former", or "current". Educational level was classified as "less than 10 years", "11-15 years", "16-20 years" or "more than 20 years". Diabetes was considered present if the participant was taking insulin or an oral hypoglycaemic agent. Hypertension was defined as systolic blood pressure $\geq 140$ or diastolic blood pressure $\geq 90 \mathrm{mmHg}$ or current use of antihypertensive medications [16]. Other covariates obtained from the parent cohorts were age, sex, race, BMI, and levels of serum triglycerides and high-density lipoprotein cholesterol.

\section{Statistical analysis}

Data is presented as mean \pm standard deviation for continuous variables and number (percentage) for categorical variables. Cox proportional hazards models were used to evaluate the association between $\mathrm{NC}$ and incident CHF or CHD mortality. The overall significance of the association of $\mathrm{NC}$ with each outcome was tested with NC modelled as a continuous variable. Survival time was defined as the time from baseline polysomnogram to the first CHF (or CHD death) event. Censoring time was the time of last known disease-free status for those without cardiovascular disease. All analyses were adjusted in the model for the following covariates: (1) age, sex, and BMI; (2) age, sex, BMI, and waist circumference; (3) age, sex, BMI, waist circumference, AHI, smoking status, serum levels of total cholesterol, triglycerides and high-density lipoprotein, history of diabetes, and history of hypertension, which are common risk factors for cardiovascular diseases. The relationship between different quartile levels of $\mathrm{NC}$ and the incidence of $\mathrm{CHF}$ or $\mathrm{CHD}$ mortality is presented as hazard ratio (HR) [95\% confidence intervals (CI)]. Net reclassification improvement (NRI) index and integrated discrimination improvement (IDI) index were calculated to assess the incremental value of $\mathrm{NC}$ in predicting cardiovascular diseases. $P$ values $<0.05$ were considered to be statistically significant. Statistical analyses were performed with SPSS software (version 22.0, IBM Corp., Armonk, NY, USA) as well as with $\mathrm{R}$ (version 3.0.1, $\mathrm{R}$ Foundation for Statistical Computing, Vienna, Austria).

\section{Results}

A total of 4433 subjects without CHD or CHF at baseline were followed up for a median of 10.9 years. It was a predominantly Caucasian population $(86.6 \%)$ with an average age of 63 years (results not displayed). The group without SDB was younger $(60.93 \pm 11.05$ years $)$ and had more female patients (67.4\%), lower BMI, and less cardiovascular risks such as dyslipidaemia, abnormal blood pressure, and diabetic status than the group with SDB $(P<0.05)$ (Additional file 2: Table S1). More baseline characteristics are shown in Additional file 3: Table S2.

During the follow-up period, $163 \mathrm{CHF}$ events and 58 CHD deaths were observed in the group with non-SDB, and 251 and 86 in the group with SDB, respectively. There was a positive linear association between $\mathrm{NC}$ quartiles and CHF incidence in the group without SDB $(P=0.027)$, while no association was observed in the SDB group. The same relationship also existed between $\mathrm{NC}$ and $\mathrm{CHD}$ mortality in the group without SDB $(P=0.003)$ (Additional file 4: Figure S2). Survival analysis also revealed the difference in the outcomes between these two subgroups. It was found that the CHD death-free curve in the group without SDB had greater separation and more obvious consistency with $\mathrm{NC}$ quartiles than the one in the SDB group. In addition, the tendency for a higher incidence of $\mathrm{CHF}$ with increasing $\mathrm{NC}$ quartiles existed in the group without SDB only (Fig. 1).

In the group without SDB, after adjustment for age, sex, and BMI, the HR for the incidence of CHF was 2.265 (95\% CI 1.074-4.777) in the highest NC quartile compared with that in the lowest NC quartile. After further adjustment for waist circumference and other variables, the association was diminished and became not significant. However, the association of NC, as a continuous variable, with $\mathrm{CHF}$ incidence remained significant with an adjusted HR of 1.082 (95\%CI 1.003-1.166) per unit increase in $\mathrm{NC}$ after even adjusting for all of the variables in the group without $\mathrm{SDB}(P=0.040)$. In the SDB group, the association was not statistically significant in both the univariate and multivariate models (Table 1 ).

Unlike the relationship between $\mathrm{CHF}$ and $\mathrm{NC}$ quartile, the HR for the incidence of fatal CHD was 5.477 (95\% CI 1.422-21.090) in the highest NC quartile compared with that in the lowest $\mathrm{NC}$ quartile after adjusting for all of the variables in the group without SDB. In addition, after adjustment for age, sex, and BMI, the HR of $\mathrm{NC}$, as a continuous variable, for $\mathrm{CHD}$ death was 1.141 (95\% CI 1.014-1.282) and $1.061(0.979-1.150)$ in the groups with and without SDB, respectively. In the model with additional adjustment for waist circumference, the association between fatal $\mathrm{CHD}$ and $\mathrm{NC}$ was significant only in the group without SDB $(P=0.022)$. With all the covariates adjusted in multivariate model 3 , there was still a significant association between $\mathrm{NC}$ and CHD death in those without SDB, with an adjusted HR of 1.134 (95\% CI 1.001-1.284) per unit increase in NC (Table 2).

To further evaluate whether $\mathrm{NC}$ has an incremental value in predicting the risk of $\mathrm{CHF}$ and CHD death, IDI and NRI were calculated in our study (Table 3). Reclassification statistics showed a significant improvement in NRI index of $0.2017(P=0.0135)$, indicating that, with 

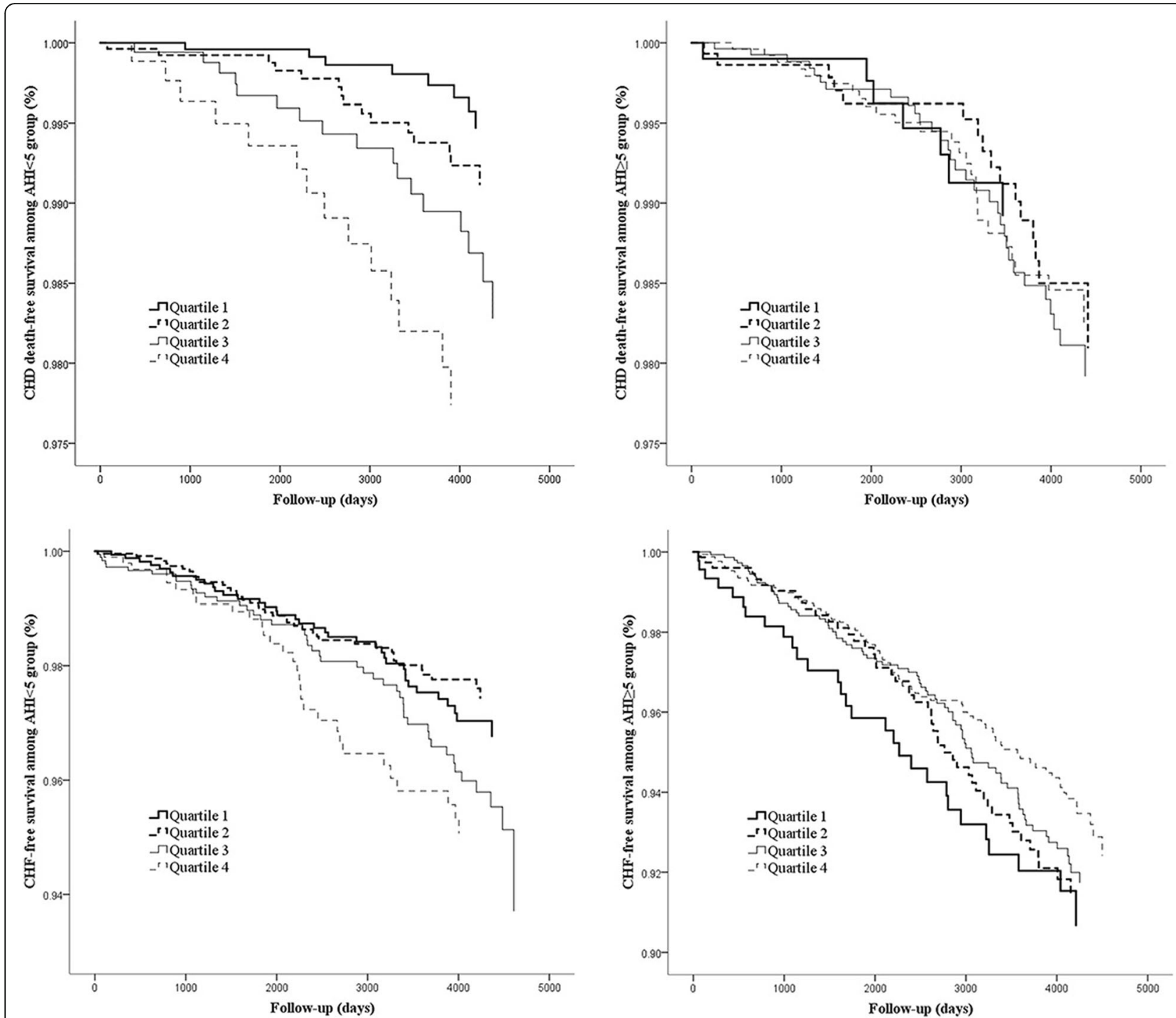

Fig. 1 Adjusted Kaplan-Meier survival curves for NC quartiles, according to the AHI group and event type. All the results were adjusted for age, sex, BMI, waist circumference, AHI, smoking status, total cholesterol, triglycerides, high-density lipoprotein, history of diabetes, and history of hypertension. $\mathrm{CHF}=$ congestive heart failure, $\mathrm{CHD}=$ coronary heart disease, $\mathrm{AHI}=$ apnoea-hypopnea index, $\mathrm{BMI}=$ body mass index, $\mathrm{NC}=$ neck circumference

the addition of $\mathrm{NC}$, the prediction power of the model adjusted for age, sex, BMI has been improved, and the proportion of correct classification increased by $20.17 \%$. The prediction for CHD death were also improved (NRI $=0.2655, P=0.0460)$. At the meantime, the IDI index was $0.0060(P=0.0447)$ in the model predicting $\mathrm{CHD}$ death in the non-SDB population, indicating that an aggregate measure of sensitivity and specificity was superior in the model adjusted for age, sex, BMI and NC compared with the one adjusted for age, sex and BMI only. However, both reclassification statistics showed no significant improvement in the predicting value with the addition of $\mathrm{NC}$ in the SDB population.

\section{Discussion}

The SHHS study was conducted in a community-based population and included ethnically diverse participants with $\mathrm{AHI}$ status. It has reported several valuable findings on the association between SDB and hypertension, CHD, and CHF [17-19]. To the best of our knowledge, our study is the first prospective cohort study to evaluate the association between $\mathrm{NC}$ and future $\mathrm{CHF}$ events and fatal CHD in a large cohort. This study indicated that participants without SDB with higher NC may develop more CHF events and CHD deaths. NC might be an early risk factor and showed preclinical predictive value for CHD death and CHF events in the population without SDB. 
Table 1 The association of incident CHF with neck circumference according to AHI categories

\begin{tabular}{|c|c|c|c|c|c|}
\hline & & Quartile & eck circumference & & \\
\hline & Q1 (low) & Q2 & Q3 & Q4 (high) & Overall tendency \\
\hline $\mathrm{AHI}<5^{\#}$ & & & & & \\
\hline Subjects, $n$ & 805 & 655 & 491 & 283 & \\
\hline Events, n (\%) & $39(4.8)$ & $45(6.9)$ & $49(10.0)$ & $30(10.6)$ & \\
\hline Univariate Model & 1 (Ref) & 1.457 (0.949-2.238) & $2.197(1.443-3.346)$ & $2.341(1.455-3.769)$ & 1.095 (1.054-1.137) \\
\hline Multivariate Model 1 & 1 (Ref) & $0.997(0.630-1.577)$ & $1.738(0.982-3.077)$ & $2.265(1.074-4.777)$ & $1.111(1.034-1.194)$ \\
\hline Multivariate Model 2 & 1 (Ref) & $0.891(0.561-1.415)$ & $1.386(0.780-2.463)$ & $1.762(0.850-3.650)$ & $1.080(1.008-1.157)$ \\
\hline Multivariate Model 3 & 1 (Ref) & $0.844(0.522-1.364)$ & 1.419 (0.778-2.589) & $1.668(0.765-3.638)$ & $1.082(1.003-1.166)$ \\
\hline $\mathrm{AHI} \geq 5^{*}$ & & & & & \\
\hline Subjects, $n$ & 304 & 455 & 681 & 759 & \\
\hline Events, n (\%) & $31(10.2)$ & $51(11.2)$ & $83(12.2)$ & $86(11.3)$ & \\
\hline Univariate Model & 1 (Ref) & $1.086(0.695-1.697)$ & $1.196(0.792-1.807)$ & 1.107 (0.734-1.669) & $1.004(0.974-1.035)$ \\
\hline Multivariate Model 1 & 1 (Ref) & $1.051(0.662-1.670)$ & $1.065(0.646-1.757)$ & $1.107(0.600-2.041)$ & 0.999 (0.948-1.054) \\
\hline Multivariate Model 2 & 1 (Ref) & $0.972(0.609-1.550)$ & $0.969(0.587-1.599)$ & $0.995(0.544-1.820)$ & $0.996(0.947-1.049)$ \\
\hline Multivariate Model 3 & 1 (Ref) & $0.870(0.533-1.418)$ & $0.807(0.480-1.357)$ & $0.690(0.364-1.309)$ & $0.965(0.912-1.022)$ \\
\hline
\end{tabular}

Model 1 adjusted for age, gender, and BMI

Model 2 adjusted for age, gender, BMI, and waist circumference

Model 3 adjusted for age, gender, BMI, waist circumference, AHI, smoking status, total cholesterol, triglycerides, high-density lipoprotein, history of diabetes, and history of hypertension

Results are presented as hazard ratio (95\% confidence interval). CHF congestive heart failure, $A H I$ apnoea-hypopnea index (the average number of episodes of apnoea and/or hypopnea per hour of sleep), BMI body mass index

${ }^{\#} P$ value for the overall tendency of neck circumference modelled as a continuous variable was $<0.001,0.004,0.029,0.040$ in the univariate model, multivariate model 1 , model 2 , and model 3 , respectively

${ }^{*} P$ value for the overall tendency of neck circumference modelled as a continuous variable was $0.809,0.978,0.893$, and 0.225 in univariate model, multivariate model 1, model 2, and model 3, respectively

Table 2 The association of CHD death with neck circumference according to AHI categories

\begin{tabular}{|c|c|c|c|c|c|}
\hline & \multicolumn{4}{|c|}{ Quartiles of neck circumference } & \multirow[b]{2}{*}{ The overall tendency } \\
\hline & Q1 (low) & Q2 & Q3 & Q4 (high) & \\
\hline \multicolumn{6}{|l|}{$\mathrm{AHI}<5^{\#}$} \\
\hline Subjects, $\mathrm{n}$ & 805 & 655 & 491 & 283 & \\
\hline Events, n (\%) & $10(1.2)$ & $17(2.6)$ & $17(3.5)$ & $14(4.9)$ & \\
\hline Univariate Model & 1 (Ref) & $2.120(0.971-4.631)$ & $2.951(1.351-6.445)$ & 4.164 (1.849-9.374) & $1.117(1.049-1.189)$ \\
\hline Multivariate Model 1 & 1 (Ref) & $1.622(0.710-3.705)$ & 2.760 (0.977-7.798) & $5.480(1.574-19.075)$ & $1.141(1.014-1.282)$ \\
\hline Multivariate Model 2 & 1 (Ref) & $1.629(0.714-3.717)$ & $2.782(0.992-7.807)$ & $5.486(1.624-18.527)$ & $1.139(1.019-1.274)$ \\
\hline Multivariate Model 3 & 1 (Ref) & $1.914(0.762-4.807)$ & $3.427(1.806-10.814)$ & $5.477(1.422-21.090)$ & $1.134(1.001-1.284)$ \\
\hline \multicolumn{6}{|l|}{$\mathrm{AHI} \geq 5^{*}$} \\
\hline Subjects, $n$ & 304 & 455 & 681 & 759 & \\
\hline Events, n (\%) & $8(2.6)$ & $18(4.0)$ & $34(5.0)$ & $26(3.4)$ & \\
\hline Univariate Model & 1 (Ref) & $1.479(0.643-3.402)$ & $1.886(0.873-4.074)$ & $1.291(0.584-2.851)$ & $1.013(0.962-1.068)$ \\
\hline Multivariate Model 1 & 1 (Ref) & $1.773(0.749-4.194)$ & $2.115(0.858-5.215)$ & $1.972(0.663-5.868)$ & $1.061(0.979-1.150)$ \\
\hline Multivariate Model 2 & 1 (Ref) & $1.643(0.690-3.914)$ & $1.891(0.762-4.690)$ & $1.735(0.586-5.135)$ & $1.050(0.968-1.139)$ \\
\hline Multivariate Model 3 & 1 (Ref) & $1.292(0.504-3.309)$ & $1.538(0.579-4.087)$ & $1.393(0.472-4.538)$ & $1.033(0.944-1.130)$ \\
\hline
\end{tabular}

Model 1 adjusted for age, gender, and BMI

Model 2 adjusted for age, gender, BMl, and waist circumference

Model 3 adjusted for age, gender, BMI, waist circumference, AHI, smoking status, total cholesterol, triglycerides, high-density lipoprotein, history of diabetes, and history of hypertension

Results are presented as hazard ratio (95\% confidence interval). CHD coronary heart disease, $A H I$ apnoea-hypopnea index, $B M I$ body mass index

${ }^{\#} P$ value for the overall tendency of neck circumference modelled as a continuous variable was $0.001,0.028,0.022$, and 0.048 in univariate model, multivariate model 1 , model 2 , and model 3 , respectively

${ }^{*} P$ value for the overall tendency of neck circumference modelled as a continuous variable was $0.616,0.149,0.236$, and 0.478 in univariate model, multivariate model 1 , model 2 , and model 3 , respectively 
Table 3 Incremental value of neck circumference in predicting the risk of incident $\mathrm{CHF}$ and $\mathrm{CHD}$ death

\begin{tabular}{llll}
\hline & & CHF & CHD death \\
\hline $\mathrm{AHI}<5$ & $\mathrm{NRI}$ & $0.2017(0.0420-0.3614)$ & $0.2655(0.0051-0.5260)$ \\
& $P$ & 0.0135 & 0.0460 \\
& $\mathrm{IDI}$ & $0.0055(-0.0008-0.0118)$ & $0.0060(0.0001-0.0119)$ \\
& $P$ & 0.0885 & 0.0447 \\
$\mathrm{AHI} \geq 5$ & $\mathrm{NRI}$ & $0.0081(-0.1233-0.1395)$ & $0.0862(-0.1288-0.3012)$ \\
& $P$ & 0.9041 & 0.4331 \\
& $\mathrm{IDI}$ & $0.0003(-0.0005-0.0012)$ & $-0.0006(-0.0031-0.0019)$ \\
& $P$ & 0.3785 & 0.6398 \\
\hline
\end{tabular}

Reclassification indices were calculated for the addition of neck circumference in the model adjusted for age, sex, and BMI

$A H I$ apnoea-hypopnea index, $B M I$ body mass index, $C H F$ congestive heart failure, $C H D$ coronary heart disease, NRI net reclassification improvement, $I D I$ integrated discrimination improvement

Body fat is mainly located beneath the skin, around the abdominal organs (stomach, liver, intestines, kidneys, etc.), within the bone marrow, and within muscles [20]. It has been reported that nearly all of the major cardiovascular risk factors, such as blood glucose level, plasma lipid ratio, and blood pressure worsen with obesity [21]. Conversely, low BMI has also been shown to be a risk factor for cardiovascular and bleeding events in a prospective observational study [22]. Therefore, BMI might be not capable of reflecting the characteristics of local fat deposition as different compartments of body fat have been demonstrated to be associated with heterogeneous physiological and pathological metabolism [23].

VAT accounts for $10-20 \%$ of the total body fat in men and $5-10 \%$ in women, and has been reported to predict cardiovascular diseases independent of traditional measures of obesity [24]. SAT, which is over $80 \%$ of the total body fat around the abdominal organs, buttocks, thighs, and neck, has drawn more attention in recent decades. These subcutaneous layers are functionally distinct and independently correlate with metabolic complications [25]. For example, gluteofemoral SAT was found to contribute to the long-term entrapment of excess fatty acids and protect from the adverse effects associated with ectopic fat deposition [26]. Additionally, several studies have reported that waist-to-hip ratio was strongly positively associated with cardiovascular outcomes in comparison with BMI [27, 28].

$\mathrm{NC}$, as an anatomically separate component of the body, is a distinct fat depot that represents the upper body SAT. The Framingham Heart Study demonstrated that $\mathrm{NC}$ was a novel measurement for cardio-metabolic risk and associated with cardiovascular disease risk factors, including blood pressure, triglycerides, and fasting blood glucose [3]. However, there was no statistically significant association between $\mathrm{NC}$ and incidence of cardiovascular disease in the entire population according to their secondary analysis, while another study found that $\mathrm{NC}$ independently contributed to the prediction of cardio-metabolic risks that may be superior to waist circumference or BMI [29]. Our study has also shown that a higher $\mathrm{NC}$ may contribute to $\mathrm{CHF}$ as well as $\mathrm{CHD}$ mortality, but only in the non-SDB population, after adjusting for BMI and other cardiovascular risk factors.

The potential mechanism may involve elevated levels of plasma FFAs [30]. The FFAs in circulation could inhibit oxidation and glucose uptake, and impair insulin signalling transduction through a variety of pathways, leading to insulin resistance. In addition, FFAs are also associated with lipid metabolism disorder, vascular endothelial injury, and hypertension [31]. Upper body SAT, typically represented by $\mathrm{NC}$, has been demonstrated to be associated with a much larger proportion of systemic FFAs [32]. Therefore, NC might be a much earlier signal of predicting cardiovascular disease (such as $\mathrm{CHF}$ or CHD death) amongst the anthropometric indicators. Additionally, as an index of upper body obesity, NC is always recognized as a simple and time saving measurement. Compared with waist circumference, $\mathrm{NC}$ is less affected by the changes in the body size caused by breathing, exercises, diet, and lifestyle habits such as drinking alcohol [33, 34]. The measurement of NC might be especially useful in special populations such as morbidly obese people, patients on bed rest, and pregnant women [34].

It should be noted that the predictive value of $\mathrm{NC}$ has not been found in individuals with $\mathrm{AHI} \geq 5$ in our study, although the baseline characteristics showed that the members of this group were older and suffered from higher BMI, NC, waist circumference, and other cardiovascular risks. It is proposed that the predictive value of $\mathrm{NC}$ level in the SDB group may be diminished by the strong association of SDB with $\mathrm{NC}$ and cardiovascular diseases. First, studies found that NC was significantly higher in patients with severe SDB than in those with non-severe SDB whether obese or not, possibly due to the increased mass of upper respiratory tract soft tissues $[35,36]$. In addition, SDB and NC were demonstrated to be associated with lower levels of high-density lipoprotein and incidence of diabetes, which may directly contribute to cardiovascular diseases [34, 37, 38]. SDB may also cause cardiovascular diseases by sympathetic nervous system activation and systemic inflammation resulting from intermittent hypoxemia [39]. Therefore, a larger cohort of SDB and non-SDB population may be needed to further investigate the interactions between multiple risk factors.

This study has certain limitations that deserve discussion. Firstly, although NC is a simple and feasible anthropometric measurement, there are other factors that influence $\mathrm{NC}$ other than fat deposition. Further imaging 
studies on quantification of SAT of the neck are required to validate our study findings. Secondly, the small number of CHD death events in the people without SDB did not exceed the number of covariates by at least ten-times, which made the multivariate model 3 less powerful. Thirdly, as in an observational analysis, residual confounding by unmeasured variables, such as diet or physical activity, cannot be excluded, despite multivariate adjustment for existing risk factors.

\section{Conclusions}

A higher NC might predict the incidence of $\mathrm{CHF}$ and CHD deaths in a community-based population without SDB. However, precise examination of the relationship between neck SAT on cardiovascular outcomes and the possible underlying mechanism is worth investigations in the future.

\section{Additional files}

Additional file 1: Figure S1. Flow chart of the study sample.

$\mathrm{AHI}=$ apnoea-hypopnea index. (TIF $1700 \mathrm{~kb}$ )

Additional file 2: Table S1. Characteristics of subjects between the population with SDB and non-SDB. (DOC $18 \mathrm{~kb}$ )

Additional file 3: Table S2. Characteristics of subjects by neck circumference quartiles. (DOC $59 \mathrm{~kb}$ )

Additional file 4: Figure S2. The relationship between NC and CHF or CHD death. There was a positive linear association between NC quartiles and $\mathrm{CHF}$ incidence or CHD mortality in the group without SDB, while no association was observed in the SDB group. The rate of outcome events was represented within each NC quartile according to $\mathrm{AHI}$ categories. $\mathrm{AHI}=$ apnoea-hypopnea index, $\mathrm{CHF}=$ congestive heart failure, $\mathrm{CHD}=$ coronary heart disease, $\mathrm{NC}=$ neck circumference. (TIF $2144 \mathrm{~kb}$ )

\section{Abbreviations}

AHI: Apnoea-hypopnea index; BMI: Body-mass index; CHD: Coronary heart disease; CHF: Congestive heart failure; Cl: Confidence interval; HR: Hazard ratio; IDI: Integrated discrimination improvement; NC: Neck circumference; NRI: Net reclassification improvement; SAT: Subcutaneous adipose tissue; SDB: Sleep-disordered breathing; SHHS: Sleep Heart Health Study; VAT: Visceral adipose tissue

\section{Acknowledgements}

We appreciate the Brigham and Women's Hospital for sharing the datasets of the Sleep Heart Health Study (SHHS). The SHHS acknowledges the Atherosclerosis Risk in Communities Study, the Cardiovascular Health Study, the Framingham Heart Study, the Cornell/Mt. Sinai Worksite and Hypertension Studies, the Strong Heart Study, the Tucson Epidemiologic Study of Airways Obstructive Diseases, and the Tucson Health and Environment Study for allowing their cohort members to be part of the SHHS and for permitting data acquired by them to be used in the study. SHHS is particularly grateful to the members of these cohorts who agreed to participate in SHHS as well. SHHS further recognizes all of the investigators and staff who have contributed to its success. A list of SHHS investigators, staff, and their participating institutions is available on the SHHS website, https://sleepdata.org/datasets/shhs.

\section{Funding}

SHHS was supported by grants (U01HL53916, U01HL53931, U01HL53934, U01HL53937, U01HL53938, U01HL53940, U01HL53941, U01HL64360) from the National Institutes of Health.

\section{Availability of data and materials}

The datasets used and/or analyzed during the current study are available from the corresponding author on reasonable request.

\section{Authors' contributions}

GW, JJZ, QG, LYP, JML, YG, BY and BJF had full access to all of the data in the study and take responsibility for the integrity of the data and the accuracy of the data analysis and they drafted the manuscript. JJZ and QG extracted data and performed the statistical analyses. The Working Group (GW, JJZ, QG, LYP, JML, YG, BY, BJF) and all authors contributed to interpretation of data, revised the article critically for important intellectual content and approved the final version of the manuscript.

\section{Ethics approval and consent to participate}

The protocol was approved by the Institutional Review Board of each participating institution (Boston University, Case Western Reserve University, Johns Hopkins University, Missouri Breaks Research, Inc., New York University Medical Center, University of Arizona, University of California at Davis, University of Minnesota - Clinical and Translational Science Institute, University of Washington) and signed informed consents were provided by the subjects.

\section{Competing interests}

The authors declare that they have no competing interests.

\section{Publisher's Note}

Springer Nature remains neutral with regard to jurisdictional claims in published maps and institutional affiliations.

\section{Author details}

${ }^{1}$ Department of Emergency Medicine, the Second Affiliated Hospital of Xi'an Jiaotong University, Xi'an 710004, China. ${ }^{2}$ Department of Cardiology, the Second Affiliated Hospital of Xi'an Jiaotong University, Xi'an, China. ${ }^{3}$ Division of Cardiology, Department of Internal Medicine, Tongji Hospital, Tongji Medical College, Huazhong University of Science \& Technology, Wuhan, China. ${ }^{4}$ Clinical Research Centre, the First Affiliated Hospital of Xi'an Jiaotong University, Xi'an, China. ${ }^{5}$ Department of Emergency Medicine, Longhua Hospital, Shanghai University of Traditional Chinese Medicine, Shanghai, China.

Received: 19 February 2018 Accepted: 23 May 2018

Published online: 31 May 2018

\section{References}

1. Fox CS, Massaro JM, Hoffmann U, Pou KM, Maurovich-Horvat P, Liu CY, et al. Abdominal visceral and subcutaneous adipose tissue compartments: association with metabolic risk factors in the Framingham heart study. Circulation. 2007;116(1):39-48.

2. Karpe F, Pinnick KE. Biology of upper-body and lower-body adipose tissuelink to whole-body phenotypes. Nat Rev Endocrinol. 2015;11(2):90-100.

3. Preis SR, Massaro JM, Hoffmann U, D'Agostino RB Sr, Levy D, Robins SJ, et al. Neck circumference as a novel measure of cardiometabolic risk: the Framingham heart study. J Clin Endocrinol Metab. 2010;95(8):3701-10.

4. Goodpaster BH, Krishnaswami S, Resnick H, Kelley DE, Haggerty C, Harris TB, et al. Association between regional adipose tissue distribution and both type 2 diabetes and impaired glucose tolerance in elderly men and women. Diabetes Care. 2003;26(2):372-9.

5. Neeland IJ, Turer AT, Ayers CR, Berry JD, Rohatgi A, Das SR, et al. Body fat distribution and incident cardiovascular disease in obese adults. J Am Coll Cardiol. 2015:65(19):2150-1.

6. Caffo B, Diener-West M, Punjabi NM, Samet J. A novel approach to prediction of mild obstructive sleep disordered breathing in a populationbased sample: the Sleep Heart Health Study. Sleep. 2010;33(12):1641-8.

7. Fitch KV, Stanley TL, Looby SE, Rope AM, Grinspoon SK. Relationship between neck circumference and cardiometabolic parameters in HIVinfected and non-HIV-infected adults. Diabetes Care. 2011;34(4):1026-31.

8. Torriani M, Gill CM, Daley S, Oliveira AL, Azevedo DC, Bredella MA. Compartmental neck fat accumulation and its relation to cardiovascular risk and metabolic syndrome. Am J Clin Nutr. 2014;100(5):1244-51.

9. Hingorjo MR, Qureshi MA, Mehdi A. Neck circumference as a useful marker of obesity: a comparison with body mass index and waist circumference. J Pak Med Assoc. 2012;62(1):36-40. 
10. Saka M, Turker P, Ercan A, Kiziltan G, Bas M. Is neck circumference measurement an indicator for abdominal obesity? A pilot study on Turkish adults. Afr Health Sci. 2014;14(3):570-5.

11. Quan SF, Howard BV, Iber C, Kiley JP, Nieto FJ, O'Connor GT, et al. The Sleep Heart Health Study: design, rationale, and methods. Sleep. 1997;20(12):1077-85.

12. Dean DA, Goldberger AL, Mueller R, Kim M, Rueschman M, Mobley D, et al. Scaling up scientific discovery in sleep medicine: the National Sleep Research Resource. Sleep. 2016;39(5):1151-64.

13. Cho NH, Oh TJ, Kim KM, Choi SH, Lee JH, Park KS, et al. Neck circumference and incidence of diabetes mellitus over 10 years in the Korean genome and epidemiology study (KoGES). Sci Rep. 2015;5:18565.

14. Redline S, Sanders MH, Lind BK, Quan SF, Iber C, Gottlieb DJ, Bonekat WH, et al. Methods for obtaining and analyzing unattended polysomnography data for a multicenter study. Sleep Heart Health Research Group. Sleep. 1998:21(7):759-67.

15. Johns MW. A new method for measuring daytime sleepiness: the Epworth sleepiness scale. Sleep. 1991;14(6):540-5.

16. Gottlieb DJ, Redline S, Nieto FJ, Baldwin CM, Newman AB, Resnick HE, et al. Association of usual sleep duration with hypertension: the Sleep Heart Health Study. Sleep. 2006;29(8):1009-14.

17. Gottlieb DJ, Yenokyan G, Newman AB, O'Connor GT, Punjabi NM, Quan SF, et al. Prospective study of obstructive sleep apnea and incident coronary heart disease and heart failure: the sleep heart health study. Circulation. 2010;122(4):352-60.

18. Chami HA, Resnick HE, Quan SF, Gottlieb DJ. Association of incident cardiovascular disease with progression of sleep-disordered breathing. Circulation. 2011;123(12):1280-6.

19. Haas DC, Foster GL, Nieto FJ, Redline S, Resnick HE, Robbins JA, et al. Agedependent associations between sleep-disordered breathing and hypertension: importance of discriminating between systolic/diastolic hypertension and isolated systolic hypertension in the Sleep Heart Health Study. Circulation. 2005;111(5):614-21.

20. Shen W, Wang Z, Punyanita M, Lei J, Sinav A, Kral JG, et al. Adipose tissue quantification by imaging methods: a proposed classification. Obes Res. 2003;11(1):5-16.

21. Lavie CJ, De Schutter A, Milani RV. Healthy obese versus unhealthy lean: the obesity paradox. Nat Rev Endocrinol. 2015;11(1):55-62.

22. Mak KH, Bhatt DL, Shao M, Haffner SM, Hamm CW, Hankey GJ, et al. The influence of body mass index on mortality and bleeding among patients with or at high-risk of atherothrombotic disease. Eur Heart J. 2009;30(7):857-65.

23. Vanderburgh PM. Fat distribution: its physiological significance, health implications, and its adaptation to exercise training. Mil Med. 1992;157(4): 189-92.

24. Mahabadi AA, Massaro JM, Rosito GA, Levy D, Murabito JM, Wolf PA, et al. Association of pericardial fat, intrathoracic fat, and visceral abdominal fat with cardiovascular disease burden: the Framingham Heart Study. Eur Heart J. 2009;30(7):850-6.

25. Smith SR, Lovejoy JC, Greenway F, Ryan D, de Jonge L, de la Bretonne J, et al. Contributions of total body fat, abdominal subcutaneous adipose tissue compartments, and visceral adipose tissue to the metabolic complications of obesity. Metab Clin Exp. 2001:50(4):425-35.

26. Manolopoulos KN, Karpe F, Frayn KN. Gluteofemoral body fat as a determinant of metabolic health. Int J Obes. 2010;34(6):949-59.

27. Lapidus L, Bengtsson C, Larsson B, Pennert K, Rybo E, Sjostrom L. Distribution of adipose tissue and risk of cardiovascular disease and death: a 12 year follow up of participants in the population study of women in Gothenburg, Sweden. Br Med J (Clinical research ed). 1984;289(6454):1257-61.

28. Larsson B, Svardsudd K, Welin L, Wilhelmsen L, Bjorntorp P, Tibblin G. Abdominal adipose tissue distribution, obesity, and risk of cardiovascular disease and death: 13 year follow up of participants in the study of men born in 1913. Br Med J (Clin Res Ed). 1984;288(6428):1401-4.

29. Zhou JY, Ge H, Zhu MF, Wang $\sqcup$, Chen L, Tan $Y Z$, et al. Neck circumference as an independent predictive contributor to cardio-metabolic syndrome. Cardiovasc Diabetol. 2013;12:76.

30. Santosa S, Jensen MD. Why are we shaped differently, and why does it matter? Am J Physiol Endocrinol Metab. 2008;295(3):E531-5.

31. Colussi G, Catena C, Dialti V, Pezzutto F, Mos L, Sechi LA. Fish meal supplementation and ambulatory blood pressure in patients with hypertension: relevance of baseline membrane fatty acid composition. Am J Hypertens. 2014;27(3):471-81.
32. Nielsen S, Guo Z, Johnson CM, Hensrud DD, Jensen MD. Splanchnic lipolysis in human obesity. J Clin Investig. 2004;113(11):1582-8.

33. Luo $Y$, Ma X, Shen $Y, X u Y$, Xiong Q, Zhang $X$, et al. Neck circumference as an effective measure for identifying cardio-metabolic syndrome: a comparison with waist circumference. Endocrine. 2017;55(3):822-30.

34. Joshipura K, Munoz-Torres F, Vergara J, Palacios C, Perez CM. Neck circumference may be a better alternative to standard anthropometric measures. J Diabetes Res. 2016;2016:6058916.

35. Ahbab S, Ataoglu HE, Tuna M, Karasulu L, Cetin F, Temiz LU, et al. Neck circumference, metabolic syndrome and obstructive sleep apnea syndrome; evaluation of possible linkage. Med Sci Monit. 2013;19:111-7.

36. Kawaguchi Y, Fukumoto S, Inaba M, Koyama H, Shoji T, Shoji S, et al. Different impacts of neck circumference and visceral obesity on the severity of obstructive sleep apnea syndrome. Obesity (Silver Spring, Md). 2011;19(2):276-82.

37. Nieto FJ, Young TB, Lind BK, Shahar E, Samet JM, Redline $S$, et al. Association of Sleep-Disordered Breathing, sleep apnea, and hypertension in a large community-based study. JAMA. 2000;283(14):1829.

38. Seicean S, Kirchner HL, Gottlieb DJ, Punjabi NM, Resnick H, Sanders M, et al. Sleep-disordered breathing and impaired glucose metabolism in normalweight and overweight/obese individuals: the Sleep Heart Health Study. Diabetes Care. 2008;31(5):1001-6.

39. Drager LF, Mcevoy RD, Barbe F, Lorenzifilho G, Redline S. Sleep apnea and cardiovascular disease. Circulation. 2017;136(19):1840-50.

\section{Ready to submit your research? Choose BMC and benefit from:}

- fast, convenient online submission

- thorough peer review by experienced researchers in your field

- rapid publication on acceptance

- support for research data, including large and complex data types

- gold Open Access which fosters wider collaboration and increased citations

- maximum visibility for your research: over $100 \mathrm{M}$ website views per year

At BMC, research is always in progress.

Learn more biomedcentral.com/submissions 